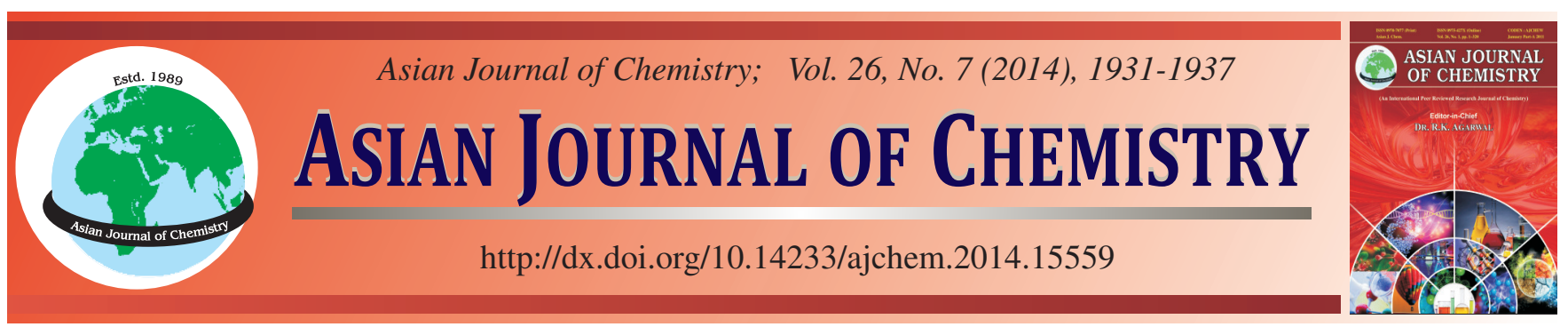

\title{
Electrochemical Behavior of Trazodone at Mercury and Glassy Carbon Electrodes and Voltammetric Methods for its Determination
}

\author{
C. KAÇAR ${ }^{*}$, Z. Durmus and E. KILIÇ
}

Department of Chemistry, Faculty of Science, Ankara University, Ankara, Turkey

*Corresponding author: Tel: + 90312 2126720; E-mail: ckacar@science.ankara.edu.tr; cerenkacar@gmail.com

\begin{abstract}
Electrochemical oxidation and reduction characteristics of trazodone were studied on glassy carbon electrode (GCE) and hanging mercury drop electrode (HMDE), respectively. Diffusion-adsorption behavior and electrochemical parameters such as diffusion coefficient, number of electrons transferred and proton participated to its electrode mechanisms and surface coverage coefficient were calculated using the results of cyclic voltammetry and square-wave voltammetry. Quasi-reversible and adsorption controlled reduction mechanism was proposed on HMDE and oxidation with two-electron/two-proton irreversible oxidation mechanism controlled by adsorption with some diffusion contribution on GCE was proposed. Experimental parameters were optimized to develop new, accurate, rapid, selective and simple voltammetric methods for direct determination of trazodone in pharmaceutical dosage forms and spiked human serum samples without time-consuming steps prior to drug assay. In square-wave cathodic adsorptive stripping voltammetry, limit of detection (LOD) was found as $4.32 \mathrm{nM}$. Proposed methods were successfully applied to determine the trazodone content of commercial pharmaceutical preparations and spiked human serum. The methods were found to be highly accurate and precise, having a relative standard deviation of less than $5 \%$ for all applications.
\end{abstract}

Keywords: Trazodone, Square-wave adsorptive stripping voltammetry, Biological samples, Hanging mercury drop electrode.

L - - - - - - - - - - - - -

\section{INTRODUCTION}

Trazodone (TRD), chemically known as 2-[3-[4-( $m$-chlorophenyl)-1-piperazinyl]-propyl]-1,2,4-triazolo-[4,3,a]pyridine$3(2 \mathrm{H}$ )-one hydrochloride (Fig. 1) is a psychoactive drug which belongs to the group of second generation of non-tricyclic antidepressants. Although its mechanism of action in humans is not clear, it is used to treat the symptoms of depression and is sometimes prescribed as a sedative and also referred to as a serotonin antagonist reuptake inhibitor (SARI) ${ }^{1,2}$.

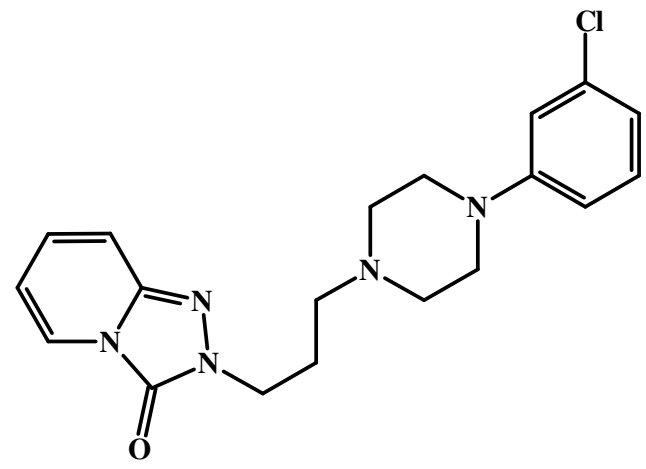

Fig.1. Chemical structure of trazodone
Many analytical methods have been described for determination of trazodone in pharmaceutical samples or biological fluids such as: trazodone selective electrodes ${ }^{3,4}$, capillary electrophoresis $^{5,6}$, high-performance liquid chromatography ${ }^{7-12}$, liquid chromatography ${ }^{13-16}$, atomic emission and atomic absorption spectrometry $^{17}$, spectrophotometry ${ }^{18-23}$, spectrofluorimetry ${ }^{24}$, GC-MS ${ }^{25}$ and voltammetry on different electrodes ${ }^{26-28}$. All chromatographic methods for the quantitation of trazodone require tedious and time consuming pretreatment such as solidphase extraction and highly sophisticated instrumentation. Spectrophotometric methods are not suitable for determination of drug molecules in biological samples. On the other hand, voltammetric techniques are used for the quantitative determination of a variety of organic and inorganic substances including drug active ingredients and excipients in pharmaceutical dosage forms and their possible metabolites in biological fluids. These techniques are also used to clarify the redox processes occuring in various working medium.

Generally, voltammetric methods are sensitive, rapid and economic when compared with chromatographic methods. It is also possible to determine various electro active species from mixtures using voltammetric methods if their voltammetric signals are separated enough. Additionally, stripping techniques 
extends the use of voltammetric methods ensuring lower detection limits. In literature, there are many applications of voltammetric stripping methods to determine environmentally and biologically important substances ${ }^{29-34}$.

Present study was designed to investigate the electrochemical reduction behavior of trazodone on hanging mercury drop electrode (HMDE) and electrochemical oxidation behavior on glassy carbon electrode (GCE). Proposing tentative reaction mechanisms on both electrodes with literature support was also aimed. Regarding these investigations, the purpose is also to develop rapid, simple and new methods for direct determination of trazodone in pharmaceutical dosage form and human plasma. For reduction peak on HMDE square-wave cathodic adsorptive stripping voltammetry (SWCAdSV) and for oxidation peak on GCE square-wave anodic adsorptive stripping voltammetry (SWAAdSV) was applied. Time consuming and expensive extraction procedures are not needed in sample preparation step.

\section{EXPERIMENTAL}

All voltammetric measurements were carried out using a $\mathrm{CH}$-instrument electrochemical analyzer (CHI 760). The three electrode cell system incorporating the platinum wire as an auxiliary electrode (BAS MW-1034), $\mathrm{Ag} / \mathrm{AgCl}$ in $3.0 \mathrm{~mol} \mathrm{~L}^{-1}$ $\mathrm{KCl}$ solution as reference electrode (MF-2052 RE-5B), hanging mercury drop electrode (HMDE BAS CGME 1108; 0.0145 $\mathrm{cm}^{2}$ ) and a glassy carbon electrode (GCE; BAS; MF 2012; $0.071 \mathrm{~cm}^{2}$ ) as working electrodes was used. Before each experiment GCE was polished manually with slurries prepared from $0.01 \mu \mathrm{m}$ aluminum oxide on a smooth polishing pad (BAS velvet polishing pad), then rinsed with double-distilled water thoroughly.

All $\mathrm{pH}$ measurements were made with Thermo Orion Model 720A pH ion meter having an Orion combined glass pH electrode (912600) (produced by Thermo Fisher Scientific). Double-distilled water was supplied from Human Power I+, Ultra Pure Water System (Produced by ELGA as PURELAB Option-S). All measurements were performed at room temperature.

Trazodone was purchased from Sigma (EC 246-855-5) and Desyrel ${ }^{\circledR}$ tablets $(50 \mathrm{mg}$ trazodone $\mathrm{HCl}$ per tablet, produced by CINAY KIMYA) as its pharmaceutical dosage form was purchased from local market in Ankara. All chemicals used were reagent grade.

Stock solutions of trazodone $\left(1 \times 10^{-3} \mathrm{~mol} \mathrm{~L}^{-1}\right)$ were prepared in double-distilled water and kept in the dark in a refrigerator. Trazodone working solutions were prepared by sufficient dilution of stock solution with supporting electrolyte and used within $24 \mathrm{~h}$ to avoid possible decomposition.

Procedure: For voltammetric measurements, known volume of trazodone solution was pipetted into $10 \mathrm{~mL}$ supporting electrolyte. Voltammetric measurements were carried out after purified nitrogen was passed through the cell for $5 \mathrm{~min}$ to remove dissolved oxygen for the first running and $30 \mathrm{~s}$ between successive runnings. For adsorptive stripping measurements, a selected accumulation potential was then applied to the electrode for a selected accumulation time period, while the solution was stirred. At the end of the accumulation time, the stirrer was stopped and a $15 \mathrm{~s}$ rest period was allowed for the solution to become quiescent. The voltammograms were then recorded by scanning the potential towards the positive direction on GCE for oxidation studies and negative direction on $\mathrm{HMDE}$ for reduction studies versus $\mathrm{Ag} / \mathrm{AgCl}\left(3 \mathrm{~mol} \mathrm{~L}{ }^{-1}\right.$ $\mathrm{KCl}$ ) electrode by applying square-wave forms.

Preparation of Desyrel ${ }^{\circledR}$ tablets: Desyrel ${ }^{\circledR}$ tablets were used as pharmaceutical dosage form which contains $50 \mathrm{mg}$ hydrochloride salt of trazodone per tablet. Ten tablets were accurately weighed and crushed to a homogeneous fine powder in a mortar and mixed well. Approximate weight of one tablet was calculated. A powder sample, equivalent to one tablet was weighed and transferred into the calibrated flask containing about $100 \mathrm{~mL}$ of double-distilled water and content of flask was sonicated for $10 \mathrm{~min}$. After standing at room temperature, volume of the flask was completed to $250 \mathrm{~mL}$ with doubledistilled water. Then, to prepare final concentration required, sample from the clear supernatant liquor was withdrawn and quantitatively diluted with the supporting electrolyte. Quantitations were performed by means of the calibration curve method from the related calibration equations.

Preparation of spiked human serum: Drug-free human serum samples are obtained from healthy volunteers were stored frozen until assay. After gentle thawing, $2 \mathrm{~mL}$ of an aliquot volume of serum sample was spiked with trazodone in BR buffer to maintain $1 \times 10^{-4} \mathrm{M}$ concentration of trazodone in serum and dissolved in acetonitrile to precipitate serum proteins. Then the mixture was vortexed for $25 \mathrm{~s}$ and then centrifuged for $10 \mathrm{~min}$ at $5000 \times \mathrm{g}$ in order to eliminate serum protein residues and $2.0 \mathrm{~mL}$ from supernatant was taken and added into supporting electrolytes to attain the total volume of $10 \mathrm{~mL}$. Sufficient volume from this solution was taken and added to voltammetric cell contains $10 \mathrm{~mL}$ of supporting electrolyte. Quantitations were performed by means of the calibration curve method from the related calibration equations.

\section{RESULTS AND DISCUSSION}

Electrochemical reduction behavior was studied on HMDE and oxidation behavior was characterized on GCE. In these studies, electrochemical behavior, diffusion and adsorption properties of trazodone were studied by using cyclic voltammetry (CV) and square-wave voltammetry (SWV).

Electrochemical behavior of trazodone on HMDE and GCE: In CV studies, a single well-defined reduction peak at HMDE at about $-1.4 \mathrm{~V}$ when $\mathrm{pH}$ is 10 and oxidation peak on GCE at about $0.8 \mathrm{~V}$ when $\mathrm{pH}$ is 7 was observed (Fig. 2). No peak was observed when only blank BR was scanned at the same conditions. Besides, anodic and cathodic peak intensities increase with increasing concentration of trazodone which may be concluded that the reduction peak on HMDE and the oxidation peak on GCE should be caused by trazodone molecules. As could be seen from Fig. 2a there is also an anodic peak at reverse scan on HMDE suggesting the reversible nature of electro reduction of trazodone. On the other hand, there is no cathodic peak at reverse scan on GCE as could be seen in Fig. $2 b$ suggesting the irreversible nature of electro oxidation of trazodone.

To investigate the electrochemical behavior of trazodone, firstly influences of scan rate on peak potential were studied for both electrodes. Peak potential was linearly shifted to more 
negative potentials with logarithm of scan rate on HMDE by obeying the equation: $\mathrm{E}_{\mathrm{p}}(\mathrm{V})=-0.04 \log \mathrm{V}-1.48\left(\mathrm{R}^{2}=0.992\right)$ (Fig 2a, inset). Shifting of peak potential with scan rate may be concluded to have an irreversible reduction mechanism, but on reverse scan there is also an oxidation peak whose potential is nearly unchanged with scan rate. Having changed the scan rate for reduction potential and having the oxidation peak should be concluded as quasi-reversible mechanism on HMDE. For this kind oxidation process on the base of linear relationship between the peak potential $\left(\mathrm{E}_{\mathrm{p}, \mathrm{a}}\right)$ and logarithm of scan rate $(\log \mathrm{v})$ the slope of the straight line is equal to $\mathrm{RT} / \mathrm{n} \alpha \mathrm{F}$ where $\alpha$ is charge transfer coefficient, $n$ is the number of electron in rate determining step and other terms are commonly known constants ${ }^{31}$.
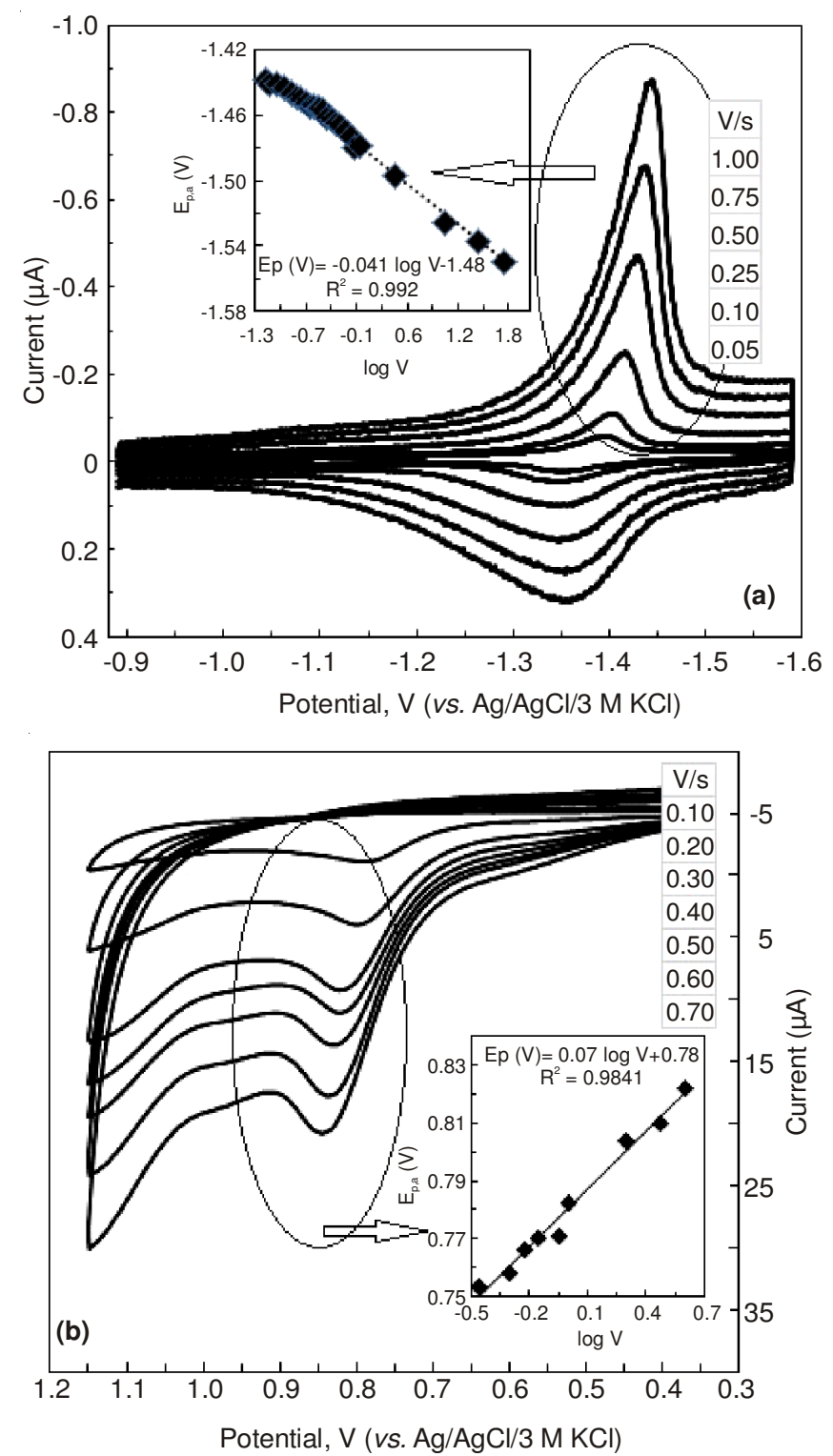

Fig. 2. Effect of scan rate on peak potential and peak current for (a) HMDE, (b) GCE (insets: relations between peak potential and logarithm of scan rate)

For reduction mechanism, value of $n \alpha$ was calculated to be 0.64 . Moreover, for irreversible electrochemical reaction, the half peak width $\left(\mathrm{W}_{1 / 2}\right)$ is equal to $62.4 / \mathrm{n}^{30,31}$. From this relation and $85-90 \mathrm{mV}$ for value of $\mathrm{W}_{1 / 2}, \mathrm{n} \alpha$ was calculated to be nearly 0.70 . Same parameter was calculated from frequency studies in $\mathrm{SWV}^{31}$ and it was found to be 0.68 . For oxidation studies on GCE, the plot of $\mathrm{E}_{\mathrm{p}, \mathrm{a}}$ versus $\log \mathrm{v}$ was found to be linear and it was expressed as $E_{p}(V)=0.07 \log V+0.78\left(R^{2}=\right.$ 0.9841) (Fig. 2b inset) and $n \alpha$ was calculated as 0.40 . For trazodone, half peak width in cyclic voltammogram was estimated to be around $120 \mathrm{mV}$, according to above equation, $n \alpha$ value was calculated as 0.52 . Same parameter was calculated from frequency studies in SWV and it was again found to be 0.45 .

Effect of scan rate on reduction and oxidation peak current on HMDE and oxidation peak current on GCE were also investigated for fixed concentration of trazodone in the 0.05-1.0 $\mathrm{Vs}^{-1}$ potential scan rate range on $\mathrm{HMDE}$ and $0.10-0.70 \mathrm{Vs}^{-1}$ on GCE. As could be seen from Fig. 3a both anodic and cathodic peak current on HMDE were linearly changed with scan rate. Cathodic peak current on HMDE obeys the relation of $i_{p, c}(\mu \mathrm{A})$ $=-2.38 \mathrm{~V}(\mathrm{~V} / \mathrm{s})-0.083$ with $\mathrm{R}^{2}=0.9948$ and current of anodic peak obtained at reverse scan of cathodic peak obeys the relation of peak $i_{\mathrm{p}, \mathrm{a}}(\mu \mathrm{A})=0.88 \mathrm{~V}(\mathrm{~V} / \mathrm{s})+0.079$ with $\mathrm{R}^{2}=$ 0.9824. On the other hand current of anodic oxidation on GCE is also found to change linearly with scan rate by obeying the equation $\mathrm{i}_{\mathrm{p}, \mathrm{a}}(\mu \mathrm{A})=1.3 \mathrm{~V}(\mathrm{~V} / \mathrm{s})+0.71$ with $\mathrm{R}^{2}=0.9857$ (Fig. $4 \mathrm{~b})$. Linear dependence of the cathodic peak current and the anodic peak current on HMDE and the anodic peak current on GCE upon the scan rate confirmed the effect of adsorption on reduction and oxidation mechanism of trazodone on both HMDE and GCE.

Plots of peak current versus square root of scan rate was also constructed and this graph is not linear even if scan rate is extremely low or extremely high for studies carried out on HMDE (Fig. 3b) and peak current changes linearly with the square-root of scan rate on GCE (Fig. 4b) indicating the inefficient effect of diffusion on mechanism to reduction and oxidation on HMDE and efficient effect to oxidation on GCE.

A plot of logarithm of cathodic peak current (A) versus logarithm of scan rate $(\mathrm{V} / \mathrm{s})$ on HMDE gave a straight line with a slope of $0.9\left(\mathrm{R}^{2}=0.9977\right)($ Fig. 3c) and for oxidation on GCE this slope was found to be $0.79\left(\mathrm{R}^{2}=0.9943\right)$ (Fig. $4 c)$. Slope value for reduction on HMDE is found to be very close to the theoretical value of 1 for adsorbed species and reduction peak on HMDE shifted to more cathodic potential with increasing concentration which confirmed that reduction process on HMDE is controlled mainly by adsorption. The slope value for GCE is less than the theoretical value for adsorption but higher than that for diffusion process which may be attributed to partial involvement of the diffusion and adsorption on electrode process.

In electrochemical studies, $\mathrm{pH}$ is one of the variables that potentially and strongly effects the reduction and/or oxidation behaviors of molecules. Effect of $\mathrm{pH}$ on peak potential and peak current were studied in CV and SWV techniques between pH 2 and 11. As could be seen from Fig. 5a, potential of reduction on HMDE shifts to more cathodic values with increasing $\mathrm{pH}$ between $\mathrm{pH}$ values of 6-11. Peak potential versus $\mathrm{pH}$, graph is linear and this linearity could be expressed as: $\mathrm{E}_{\mathrm{p}}(\mathrm{V})=$ $-0.053 \mathrm{pH}-0.75\left(\mathrm{R}^{2}=0.9944\right)$. But at $\mathrm{pH}$ values lower than 6 

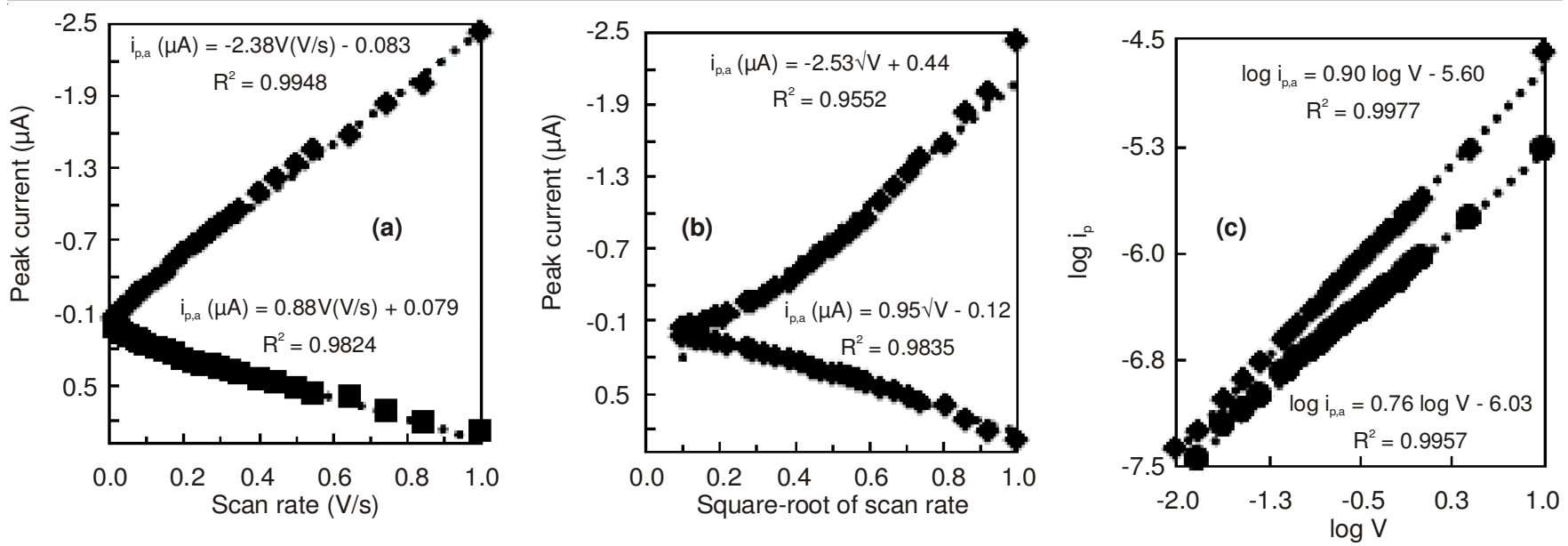

Fig. 3. Variances of peak current with scan rate on HMDE
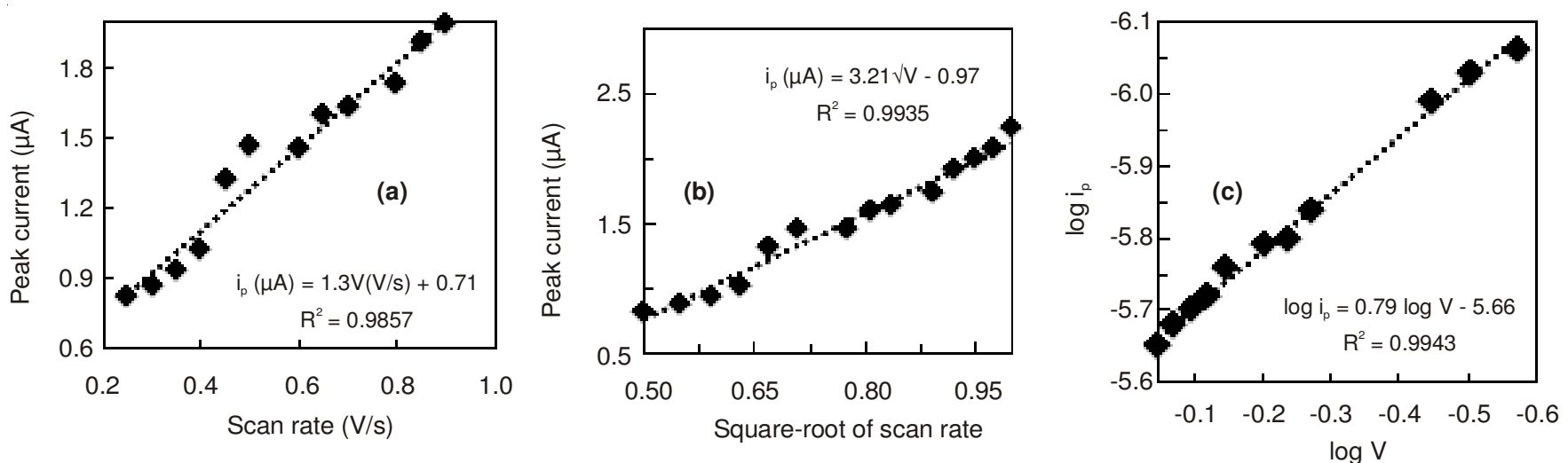

Fig. 4. Variances of peak current with scan rate on GCE

reduction peak disappeared. This behavior may be explained by two possibilities: First, at lower $\mathrm{pH}$ values (in acidic solutions) trazodone is not an electroactive molecule on HMDE, second alternative is: trazodone is an electroactive molecule in both acidic and alkaline solutions but in acidic media, peak potential is more cathodic than hydrogen over-potential on HMDE.

Slope of these $\mathrm{E}_{\mathrm{p}} v s$. $\mathrm{pH}$ should be equal to 2.303RT $\delta / \mathrm{nF}$ where $\delta$ is the number of proton involved in electrode reaction, $\mathrm{n}$ is the number of electrons transferred in electrode reaction and other terms are commonly known constants ${ }^{31}$. From these results, ratio of number of proton to number of electron was calculated to be 0.90 for HMDE, indicating the participitation of the same number of proton and electron in mechanism.

On the other hand, potential of oxidation peak on GCE was also affected by $\mathrm{pH}$ (Fig. 5b); potential versus $\mathrm{pH}$ graph is nearly linear at $\mathrm{pH}$ ranges from 5 to 9 . In this $\mathrm{pH}$ range peak
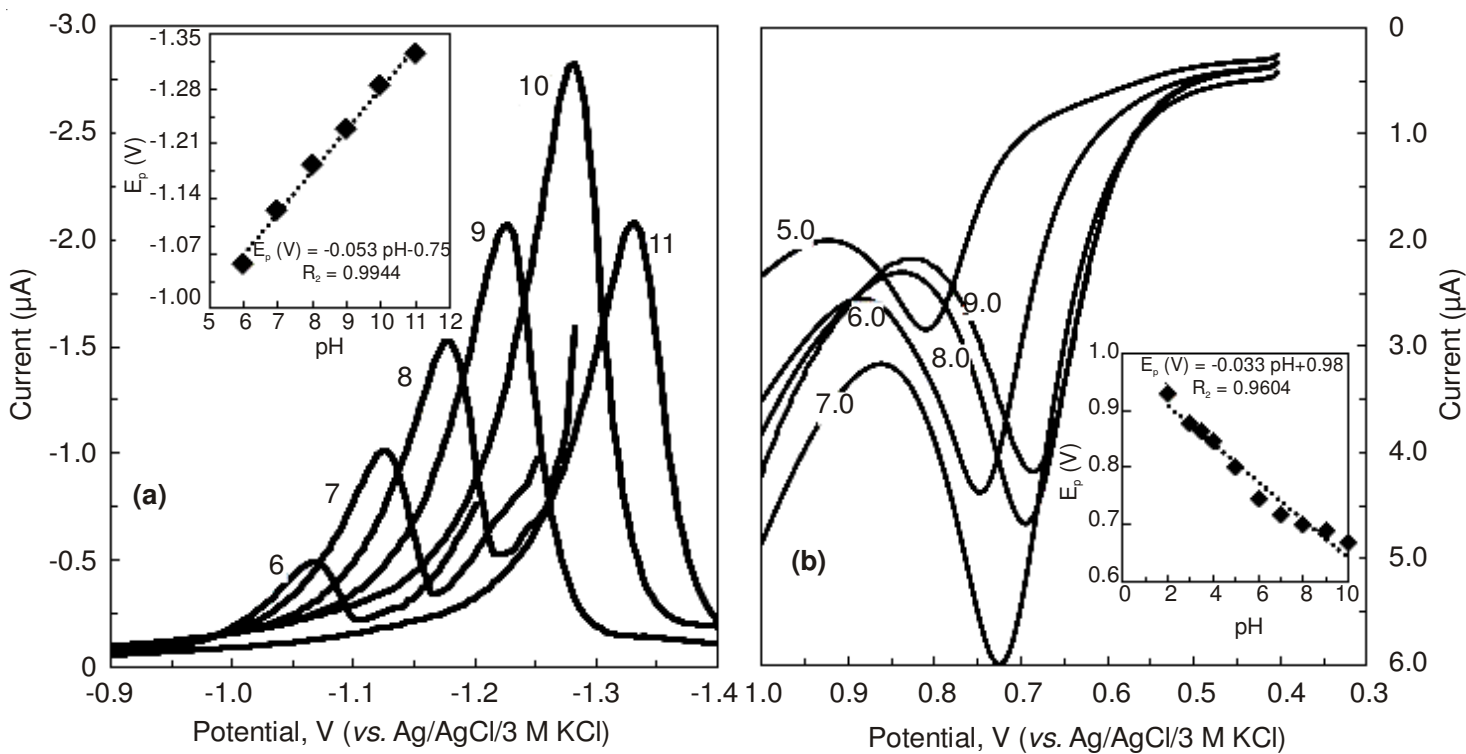

Fig. 5. Effect of $\mathrm{pH}$ on peak parameters on (a) HMDE, (b) GCE 
potential change with $\mathrm{pH}$ by obeying the correlation of: $\mathrm{E}_{\mathrm{p}}(\mathrm{V})$ $=-0.033 \mathrm{pH}+0.98\left(\mathrm{R}^{2}=0.9604\right)$. From this relation $\delta / \mathrm{n}$ value could be calculated as 0.56 .

In mechanistic studies at HMDE, to find out the numbers of electron transferred in electrode reaction (n), equations given in references ${ }^{30,31}$ were used in $\mathrm{CV}$ results and number of electrons in electrochemical step was found as $1.96 \pm 0.1$.

The surface coverage of adsorbed substance $(\Gamma)$ was calculated from the slope of curve of peak current (A) versus scan rate $(0.05-1.00 \mathrm{~V} / \mathrm{s})$ according to equation given in the same references and it was found as $6.8 \times 10^{-11} \mathrm{~mol} / \mathrm{cm}^{2}$ for reduction process on HMDE and $7.1 \times 10^{-12} \mathrm{~mol} / \mathrm{cm}^{2}$ for oxidation process on GCE.

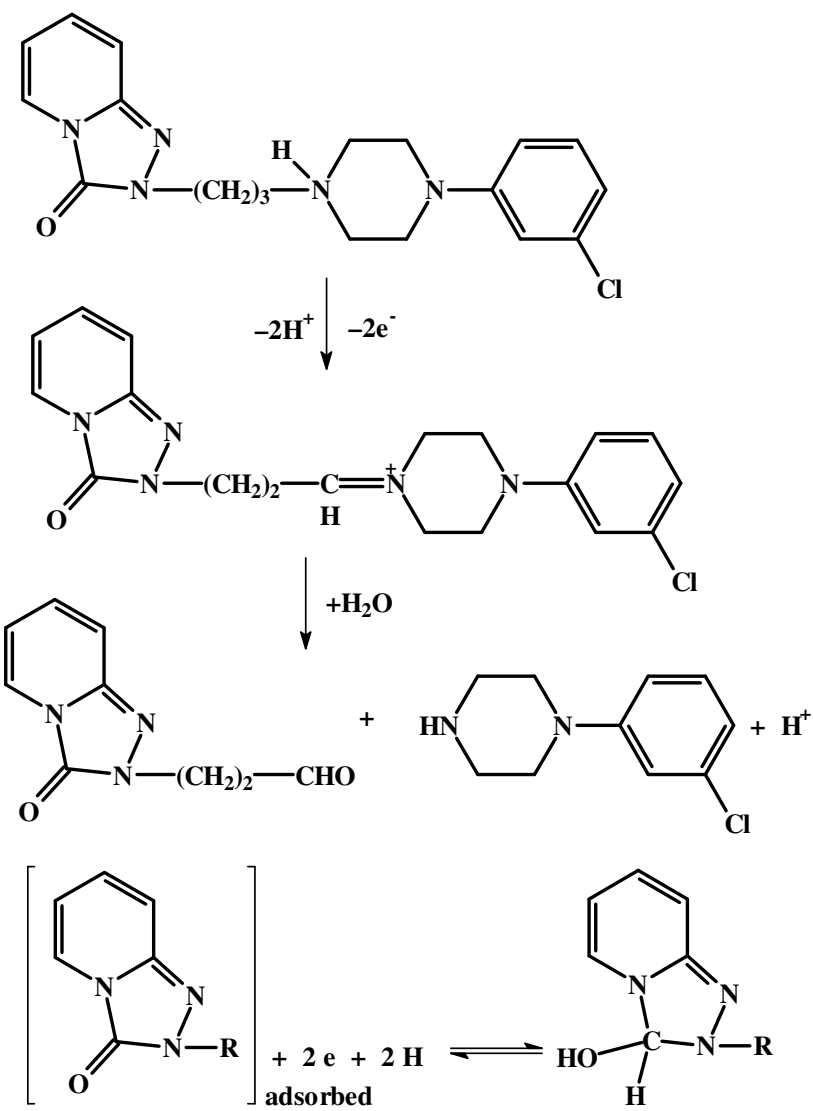

Scheme-I:Proposed oxidation and reduction mechanism for trazodone on GCE and HMDE, respectively

\begin{tabular}{lr} 
& CALIBRATION CHARACTERI \\
\hline Parameters & SW \\
\hline Linearity range (nM) & 8 \\
Slope of calibration (AL/mol) & \\
Intercept (nA) & \\
Standard deviation (SD) of regression (nA) & \\
SD of slope (AL/mol) & \\
SD of intercept (nA) & Limit of detection (nM) \\
Limit of quantification (nM) & \\
Determination coefficient (R $\left.{ }^{2}\right)$ \\
Repeatability of peak current, RSD (\%) \\
Reproducibility of peak current, RSD (\%) \\
Repeatability of peak potential, RSD (\%) \\
Reproducibility of peak potential, RSD (\%) \\
\hline
\end{tabular}

According to these results and results given in literature in similar studies for trazodone ${ }^{26-28}$ mechanisms at Scheme-I, reduction on HMDE and oxidation on GCE, may be proposed.

Voltammetric determination of trazodone: In order to develop voltammetric methods, quantitation of peak current resulting from the electroreduction of trazodone on HMDE and electrooxidation on GCE were examined using squarewave cathodic adsorptive stripping voltammetry (SWCAdSV) technique on HMDE and square-wave anodic adsorptive stripping voltammetry (SWAAdSV) on GCE. In method development studies first of all variation of peak current and its shape with instrumental conditions such as frequency (f), scan increment $\left(\Delta \mathrm{E}_{\mathrm{i}}\right)$, pulse amplitude $\left(\Delta \mathrm{E}_{\mathrm{a}}\right)$, accumulation time $\left(\mathrm{t}_{\mathrm{acc}}\right)$ and accumulation potential $\left(\mathrm{E}_{\mathrm{acc}}\right)$ was optimized. The optimization studies were carried out for $0.75 \mu \mathrm{M}$ trazodone in a BR buffer of $\mathrm{pH} 10.0$ on HMDE and $0.53 \mu \mathrm{M}$ in a BR buffer of $\mathrm{pH} 6.0$ on GCE. As a result, optimum parameters for cathodic peak were found as follows: $\mathrm{f}=25 \mathrm{~Hz}, \Delta \mathrm{E}_{\mathrm{a}}=65 \mathrm{mV}$ and $\Delta \mathrm{E}_{\mathrm{i}}$ $=2 \mathrm{mV}$; besides, the optimized parameters for anodic peak on GCE were found as follows: $\mathrm{f}=15 \mathrm{~Hz}, \Delta \mathrm{E}_{\mathrm{a}}=50 \mathrm{mV}$ and $\Delta \mathrm{E}_{\mathrm{i}}$ $=3 \mathrm{mV}$ with accumulation mode.

Adsorptive stripping techniques were optimized on both HMDE and GCE and the effect of accumulation time $\left(\mathrm{t}_{\mathrm{acc}}\right)$ and accumulation potential $\left(\mathrm{E}_{\mathrm{acc}}\right.$ ) were found as $120 \mathrm{~s}$ and $-0.45 \mathrm{~V}$, respectively for HMDE and these values found to be as $0.40 \mathrm{~V}$ and $90 \mathrm{~s}$ for GCE.

Using these optimized conditions, the applicability of the proposed procedures for determination of trazodone was examined. To obtain the calibration characteristics, peak current were measured as function of concentration at least five times and average of measurements for each trazodone concentration was used as a peak current. Graphs were given in Fig. 6 and characteristics of calibration were given in Table-1.

Validation of the proposed methods for the determination of trazodone was done by evaluation of linearity, limit of detection (LOD), limit of quantification (LOQ), repeatability (within-day), reproducibility (between-day), specifity, recovery, precision and accuracy.

The good linearity of the calibration graphs and the negligible scatter of the experimental points are clearly evident from the values of the determination coefficients and standard deviations (Table 1) for proposed methods.

LOD and LOQ values for trazodone were calculated using the relations: $\mathrm{LOD}=3 \mathrm{~s} / \mathrm{m}$ and $\mathrm{LOQ}=10 \mathrm{~s} / \mathrm{m}^{31}$. Where $\mathrm{s}$ is the

\section{TABLE-1}

\begin{tabular}{cc} 
SWCAdSV & SWAAdSV \\
\hline $8.0-610.0$ & $120.0-1250.0$ \\
0.62 & 0.21 \\
7.11 & 10.0 \\
2.06 & 41.5 \\
0.003 & 0.005 \\
0.89 & 2.66 \\
4.32 & 41.8 \\
14.4 & 126.7 \\
0.999 & 0.995 \\
4.38 & 6.72 \\
6.41 & 9.75 \\
0.93 & 1.28 \\
1.97 & 1.75 \\
\hline
\end{tabular}



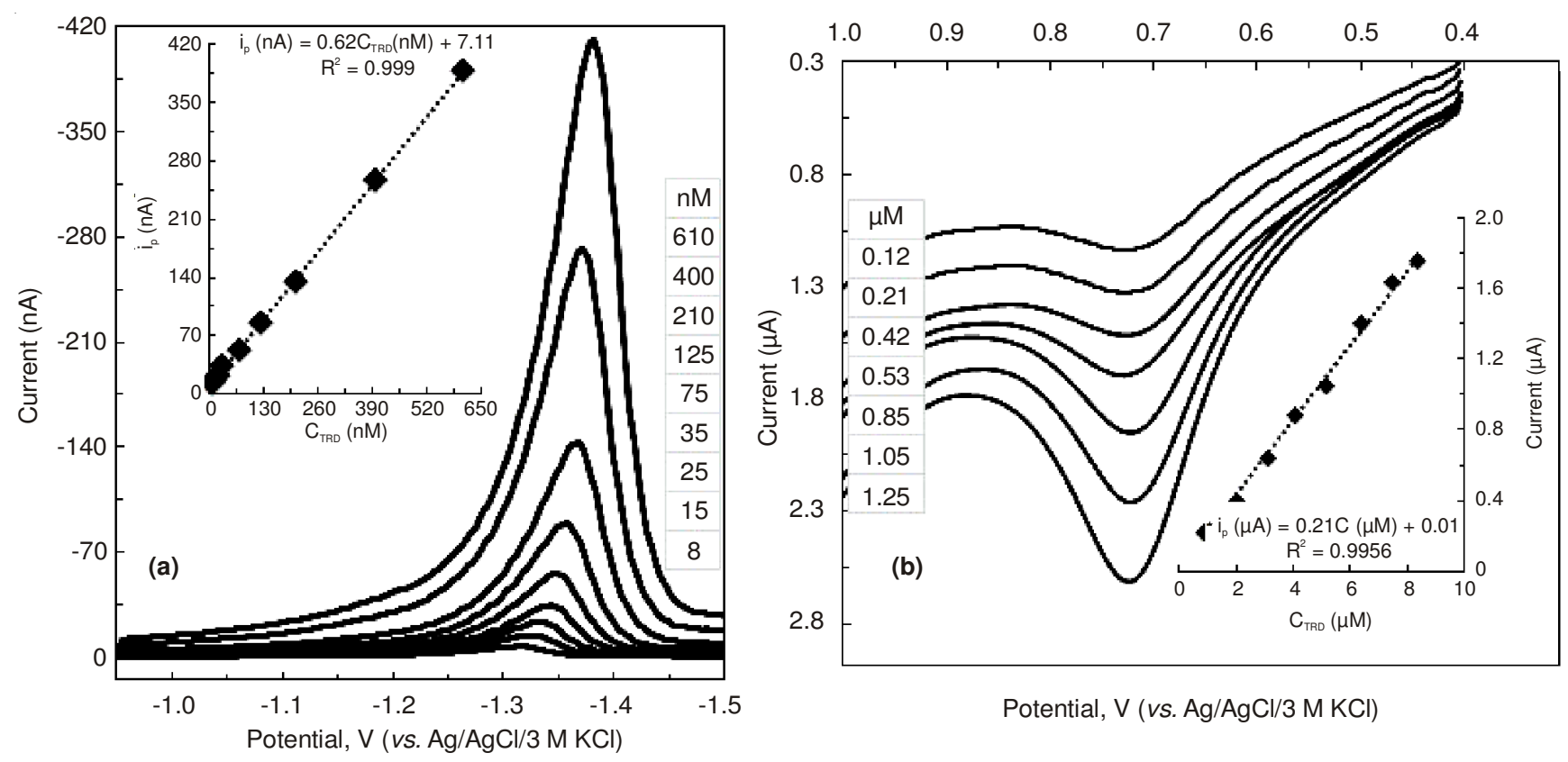

Potential, $\mathrm{V}$ (vs. Ag/AgCl/3 M KCl)

Fig. 6. Calibration voltammograms of trazodone on (a) HMDE and (b) GCE (insets: Calibration graphs)

standard deviation of intercept of calibration curve and $\mathrm{m}$ is the slope of the related calibration curve. LOD and LOQ values for each proposed methods are given in Table-1.

Trazodone assay in tablets and human serum: In order to evaluate the applicability of the proposed methods to pharmaceutical preparations and biological samples, trazodone was determined using Desyre ${ }^{\circledR}$ tablets and spiked human serum samples by using direct calibration method. When a portion of the tablet solution was added to BR at optimum $\mathrm{pH}$ value, a cathodic peak around $-1.5 \mathrm{~V}$ and an anodic peak around $0.9 \mathrm{~V}$ were recorded. Trazodone content of solution was calculated from, peak current and related calibration curve and results are presented in Table- 2 for tablet solutions and given in Table- 3 for spiked serum samples.

As could be seen from Table-2, the mean results of the applications with all techniques for both electrodes were found very close to the declared value of $50 \mathrm{mg} \mathrm{HCl}$ salt of trazodone per tablet. In order to compare the precisions and evaluate the difference of proposed methods, tablet analysis results were examined by using $\mathrm{F}$ and student $\mathrm{t}$ tests. According to F-test results the variances between methods were found to be insignificant at $95 \%$ confidence level indicating that no significant differences exist between the performances of the proposed methods regarding their precision. Besides, according to t test results it is easily to be concluded for all proposed methods that there is no significant difference between the founded and labeled trazodone amounts. These results indicate that the trazodone content of pharmaceuticals could be safely determined by using these methods without interference from other substances present in the tablet.

Besides, recovery studies in spiked human serum samples were performed by using direct calibration method. In these applications firstly voltammetric base line for trazodone-free serum samples in BR solution were taken and it was found that there is no voltammetric signal in the potential range in which studies for trazodone were carried out. It was concluded from this investigation that there is no any interference effect of any possible species found in human serum. As could be seen in Table-3, recovery values in applications to spiked serum were found to be range between $99.7 \%$ and $100.1 \%$ and differences between spiked concentration and calculated concentration by using related calibration equation are insignificant at $95 \%$ confidence level. Precision of measurements for serum samples are in good agreement since RSD values are less than $7 \%$.

These results showed that the proposed methods could be applied to trazodone assay in tablet dosage form and human serum without any pretreatment prior to analysis.

TABLE-2

RECOVERY VALUES OF PROPOSED METHODS FOR DESYREL TABLETS

\begin{tabular}{ccccc}
\hline Method & Amount labeled $(\mathrm{mg})$ & Amount found $(\mathrm{mg})$ & Mean Recovry $(\%)$ & RSD $(\%)$ \\
\hline SWCAdSV & 50 & $44.5 ; 47.2 ; 49.6 ; 52.3 ; 54.5$ & $99.2 \pm 9.9$ & 7.9 \\
SWAAdSV & 50 & $48.6 ; 49.9 ; 49.4 ; 50.9 ; 50.3$ & $99.8 \pm 1.6$ & 3.5 \\
\hline
\end{tabular}

TABLE-3

RECOVERY VALUES OF PROPOSED METHODS FOR SPIKED SERUM

\begin{tabular}{ccccc}
\hline Method & Amount spiked $(\mu \mathrm{g})$ & Amount found $(\mu \mathrm{g})$ & Mean Recovry $(\%)$ & RSD $(\%)$ \\
\hline SWCAdSV & 12.5 & $12.2 ; 12.5 ; 12.3 ; 12.7 ; 12.6$ & $99.76 \pm 1.6$ \\
SWAAdSV & 12.5 & $12.4 ; 12.9 ; 12.0 ; 12.6 ; 12.0$ & $100.12 \pm 3.6$ \\
\hline
\end{tabular}




\section{Conclusion}

Electrochemical characteristics of trazodone on HMDE and GCE were studied. To understand the mechanism of action for drug molecules and target/related organs, redox properties and electrochemical parameters could be meaningful. Determination of drug molecules themselves or any related species from serum and any other biological samples after various time of inhaling may also be important. The proposed methods for trazodone are sensitive, precise, accurate and rapid enough to be used in routine analysis. In addition, no sophisticated instrumentation like HPLC and prior tedious extraction process is required. Furthermore, percentage of recovery results indicates that the developed methods can be applied for quantitation of trazodone without interference from other ingredients.

There is no significant difference between the detection limits of proposed methods and reported chromatographic and spectrophotometric methods. Adsorptive stripping methods could be applied to the detection of trazodone in tablet dosage form. Besides, it could be stated that these methods may be more suitable for the determination of trazodone in biological medium where the detection of lower concentration is required with an insignificant matrix effect.

\section{REFERENCES}

1. E.F. Coccaro and L.J. Siever, J. Clin. Pharmacol., 21, 241 (1985).

2. R.N. Brogden, R.C. Heel, T.M. Speight and G.S. Avery, Drugs, 21, 40 (1981).

3. M.S. García, J. Ortuño, M.I. Albero and M. Cuartero, Anal. Bioanal. Chem., 394, 1563 (2009).

4. R. Ammar, N. Khalaf and A. Al-Warthan, J. Incl. Phenom. Macrocycl. Chem., 69, 287 (2011).

5. M. Himmelsbach, C.W. Klampfl and W. Buchberger, J. Sep. Sci., 28 , 1735 (2005).

6. M. Himmelsbach, W. Buchberger and C.W. Klampfl, Electrophoresis, 27, 1220 (2006)

7. G.T. Vatassery, L.A. Holden, D.K. Hazel and M.W. Dysken, Clin. Biochem., 30, 149 (1997).

8. J.J. Berzas, C. Guiberteau, A.M. Contento and V. Rodríguez, Chromatographia, 56, 545 (2002).

9. H. Kirchherr and W.N. Kühn-Velten, J. Chromatogr. B Analyt. Technol. Biomed. Life Sci., 843, 100 (2006).
10. L. Mercolini, C. Colliva, M. Amore, S. Fanali and M.A. Raggi, J. Pharm. Biomed. Anal., 47, 882 (2008).

11. X. Kang, C. Pan, Q. Xu, Y. Yao, Y.Wang, D. Qi and Z. Gu, Anal. Chim. Acta, 587, 75 (2007).

12. K.A. Johnson, X. Liu, S. Huang, V. Roongta, W.G. Humphreys and Y.Z. Shu, Anal. Methods, 2, 1542 (2010).

13. T. Shinozuka, M. Terada, E. Tanaka, Forensic Sci. Int., 162, 108 (2006).

14. B.N. Patel, N. Sharma and M. Sanyal, P.S. Shrivastav, J. Chromatogr. B Analyt. Technol. Biomed. Life Sci., 871, 44 (2008).

15. S. Carda-Broch, M.T. Gil-Agusti, L1. Monferrer-Pons and J.S. EsteveRomero, J. Chromatogr. A, 1156, 254 (2007).

16. M. Cruz-Vera, R. Lucena, S. Cárdenas and M. Valcárcel, Anal. Bioanal. Chem., 391, 1139 (2008).

17. S. Khalil and M.A. El-Ries, J. Pharm. Biomed. Anal., 27, 117 (2002).

18. R.S. Kumar, D.H. Manjunatha, S.M.T. Shaikh, J. Seetharamappa and K. Harikrishna, Chem. Pharm. Bull. (Tokyo), 54, 968 (2006).

19. K. Harikrishna, R.S. Kumar, J. Seetharamappa and D.H. Manjunatha, J. Serb. Chem. Soc., 71, 829 (2006).

20. A. El-Gindy, B. El-Zeany, T. Awad and M.M. Shabana, J. Pharm. Biomed. Anal., 26, 211 (2001).

21. F.A.F. Nour El-Dien, G.G. Mohamed and N.A. Mohamed, Spectrochim. Acta A, 65, 20 (2006)

22. G.G. Mohamed, F.A. Nour El-Dien, S.M. Khalil and N.A. Mohamed, Spectrochim. Acta A, 65, 1221 (2006).

23. M.M. Ayad, A. Shalaby, H.E. Abdellatef and M.M. Hosny, Anal. Bioanal. Chem., 376, 710 (2003).

24. G.-J. Yang, P. Liu, X.-L. Qu, Ming-Shen, C.-Y. Wang, Q.-S. Qu, X.-Y. Hu and Z.-Z. Leng, Anal. Lett., 40, 151 (2007).

25. S.M.R. Wille, P. Van Hee, H.M. Neels, C.H. Van Peteghem and W.E. Lamber, J. Chromatogr. A, 1176, 236 (2007).

26. D. Dogrukol-Ak, V. Zaimoglu and M. Tunçel, Eur. J. Pharm. Sci., 7, 215 (1999).

27. N. EL-Enany, F. Belal and M.S. Rizk, J. Pharm. Biomed. Anal., 30, 219 (2002).

28. R.N. Hegde, N.P. Shetti and S.T. Nandibewoor, Talanta, 79, 361 (2009).

29. O. Çakirer and I.H. Tasdemir, Asian J. Chem., 22, 6353 (2010).

30. F. Öztürk, I.H.Tasdemir, Z. Durmus and E. Kiliç, Coll. Czech. Chem. Commun., 75, 685 (2010).

31. I.H.Tasdemir, M.A. Akay, N. Erk and E. Kiliç, Electroanalysis, 22, 2101 (2010).

32. I.H.Tasdemir, O. Çakirer, N. Erk and E. Kiliç, Coll. Czech. Chem. Commun., 76, 159 (2011).

33. D.A. Erdogan, I.H.Tasdemir, N. Erk and E. Kiliç, Coll. Czech. Chem. Commun., 76, 423 (2011).

34. F. Öztürk, I.H.Tasdemir, D.A. Erdogan, N. Erk and E. Kiliç, Acta Chim. Slov., 58, 830 (2011). 